\title{
Deconvolution using thin-plate splines
}

\author{
U. von Toussaint, and S. Gori \\ Max-Planck-Institut für Plasmaphysik, EURATOM Association, Boltzmannstr. 2, 85748 Garching, Germany
}

\begin{abstract}
The ubiquitous problem of estimating 2-dimensional profile information from a set of line integrated measurements is tackled with Bayesian probability theory by exploiting prior information about local smoothness. For this purpose thin-plate-splines (the 2-D minimal curvature analogue of cubic-splines in 1-D) are employed. The optimal number of support points required for inversion of 2-D tomographic problems is determined using model comparison. Properties of this approach are discussed and the question of suitable priors is addressed. Finally, we illustrated the properties of this approach with 2-D inversion results using data from line-integrated measurements from fusion experiments.
\end{abstract}

PACS numbers: 42.30.W, 02.50.F, 02.50.P

\section{INTRODUCTION}

The problem of the reconstruction of a two-dimensional emission profile from line-integrated measurements is an ubiquitous one. In the original paper by Radon [1] it was shown that the reconstruction can be done by means of the Radon transform. However the presence of measurement noise and limited amounts of data prevent a straightforward deconvolution of the lineintegrated measurements, leading to meaningless results. Therefore a large number of regularized methods for the inversion procedure have been proposed [2-5]. In most cases the profile to be reconstructed is expanded in orthogonal function systems up to a given order and adapted to predefined boundary conditions. These approaches give reasonable results - provided the profile to be reconstructed is sufficiently symmetric. In irregular geometries or for localized intensity peaks the required expansion order is too large to provide regularization. However, examples from the field of fusion research which display these properties are soft $\mathrm{X}$ ray imaging systems and the bolometry diagnostic. Here we propose to exploit the favorable property of local smoothness of minimal curvature surfaces together with an adaptive distribution of support points to tackle the underdetermined and ill-posed inversion problem.

\section{TOMOGRAPHIC DIAGNOSTICS IN FUSION EXPERIMENTS}

a. Bolometry The precise measurement of power balance in fusion experiments has always been of utmost importance for a understanding of particle and energy transport. Presently, only one technique for measuring the total radiated power is widely used in fusion experiments with magnetically confined plasma: Bolometry. Usually the temperature change induced by the plasma radiation is detected using the temperature dependent conductivity of small wires attached to an absorbing foil exposed to the plasma. Recently also silicon photodiodes have been used which have the advantage of a much better time resolution of about $10^{-6} s$ compared to $2 * 10^{-5} s$ of foil based systems but on the expense of sensitivity. The sensor arrays are located a few $\mathrm{mm}$ behind precision pinholes, so that each detector is detecting a defined conic section of the plasma. The signal level of the detectors is around $10^{-5} \mathrm{~A}$ for $1 \mathrm{MW}$ total radiated power [6] and approximately 100 lines of sight are available for deconvolution. A typical setup is shown in Fig. 1a.

b. Soft-X-Ray The $\mathrm{X}$ ray emission from a plasma yields informations on a variety of parameters such as temperature, density and impurities. Since typical plasmas of interest are optically thin in the soft $\mathrm{X}$ ray region of the emission spectrum, reabsorption of the emitted radiation can be neglected. A typical set-up (the one shown in Fig. 1b is a setup developed for W7-X) consists of several sets of imaging diode arrays. Every detector diode, usually ion-implanted silicon diodes, samples radiation from a plasma region located in the viewing cone formed by the diode and the collimator aperture. One advantage of the Soft Xray diagnostic is the combination of a good spatial resolution (large number of viewing chords) with a very good time-resolution (sampling rate $i 0^{6} \mathrm{~s}^{-1}$ ). Therefore the soft X-ray diagnostic provides important informations about the magnetohydrodynamic phenomena in fusion experiments.

\section{THE TOMOGRAPHY PROBLEM}

To calculate spatial profiles from the line integrated measurements the inversion of the Radon transform is required. However the direct inversion will not provide useful profiles because the measured data contains noise and the amount of data is often very limited. Consider the following discretized tomography model where $\underline{s}$ is a K-dimensional vector of observed signals recorded 

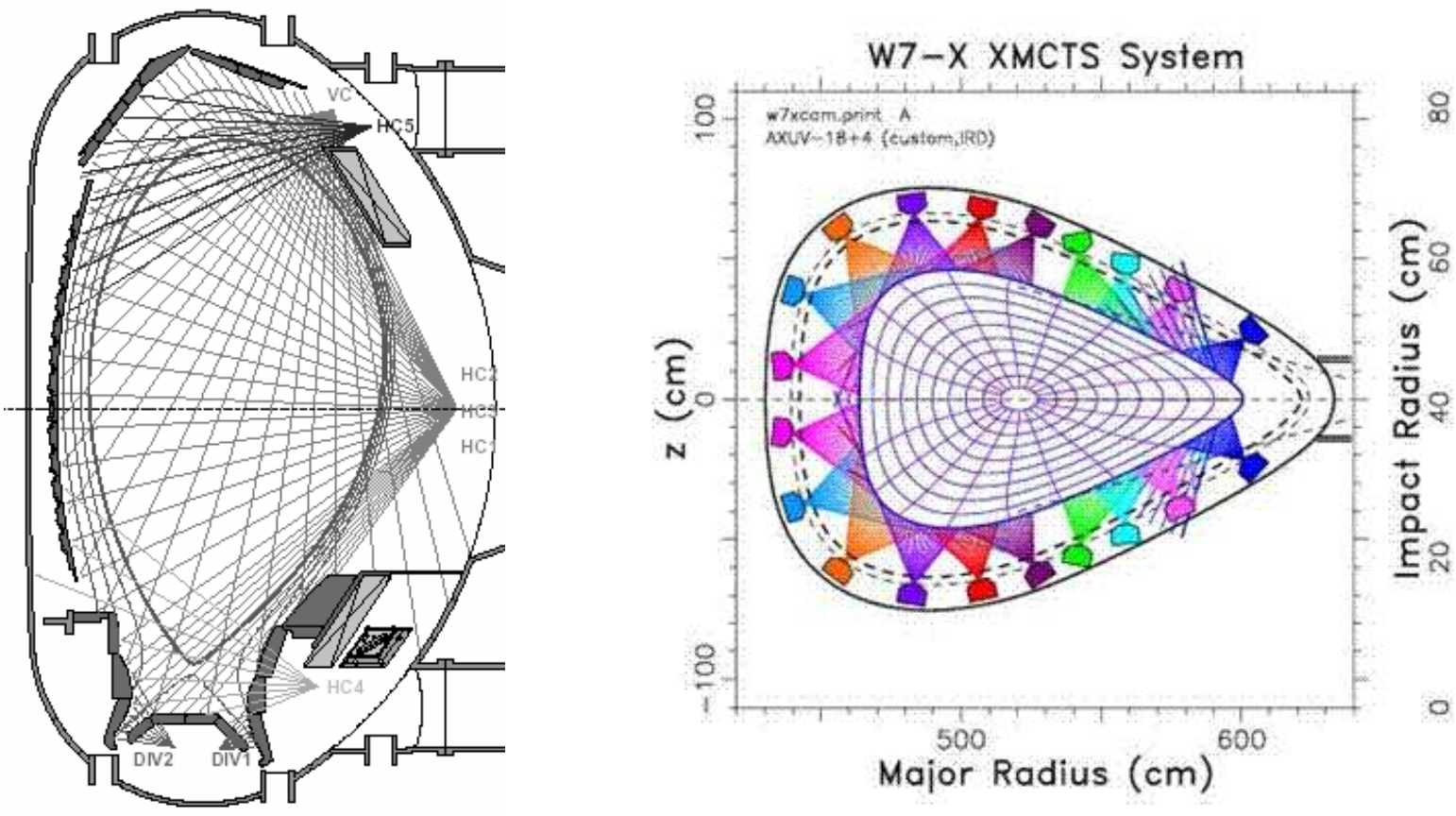

FIG. 1: a) Sensor configuration of the bolometry line integrating sensor arrays at ASDEX Ugrade(left). b) On the right hand side the intended design of the sensor configuration of the soft $\mathrm{X}$-ray line integrating sensor arrays at W7-X is shown.

by detector $\mathrm{k}$ :

$$
s_{i}=O_{i}[E]=\sum_{j} Q_{i j} E_{j}+\varepsilon_{i}
$$

where $Q$ is a (IxJ) known matrix of the proportion of the emission $E_{j}$ accumulated in detector i, $\underline{E}$ is a vector of $\mathbf{J}$ unknown emission intensities to be recovered with the property that $E_{j}>0$, and the signal recorded by sensor i is distorted by Gaussian noise $\varepsilon_{i}$. The objective is to recover $\underline{E}$ from the noisy data $\underline{s}$. Since the number of unknowns $\mathrm{J}$ outnumbers the number of data points $\mathrm{K}$ this problem is underdetermined and a regularization criterion is needed to reduce the problem to a well-posed one. In Ertl et al[7] the classical maximum-entropy formalism was chosen and subsequently Golan and Dose[8] developed a generalized maximum-entropy based approach. However, in many tomographic problems there is additional prior information available: The profile to be reconstructed is locally smooth - it exhibits local spatial correlations. These properties are explicitly not captured by entropic priors which are invariant under permutations. Therefore we propose a different approach based on the property of local smoothness.

\section{THIN-PLATE SPLINES}

A thin plate spline is the function $f(\underline{x}), \underline{x} \in R^{d}$ that minimises the curvature on a domain $\Omega([9])$ :

$$
\int_{\Omega} d \underline{x} \sum_{|\underline{v}|=2}\left(\begin{array}{l}
2 \\
\underline{v}
\end{array}\right)\left(D^{\underline{v}} f(\underline{x})\right)^{2},
$$

where $\underline{v}=\left(v_{1}, \ldots, v_{d}\right)$ is a dimensional multi-index, and $|\underline{v}|=\sum_{i=1}^{d} v_{i}$. In two dimensions $(d=2)$ the thin plate spline (TPS) can also be considered as generalization of the interpolating cubic spline in one dimension [10]. It is a commonly used basis function for modeling smooth coordinate transformations in computer vision and morphing applications. We indicate the TPS with $t(\underline{r})$, where $\underline{r}=(x, y)$ corresponds to the position on the grid in the detector field. The exposition of the basic thin-plate spline theory is along the lines of Guglielmetti[11]. The shape of the interpolating TPS surface will be given by the minimum curvature condition of eq.2. Often radial basis functions are used to represent $\mathrm{f}$ as they allow an analytical solution. More specifically, the TPS is a weighted sum of translations of radially symmetric basis functions augmented by a linear term (see 


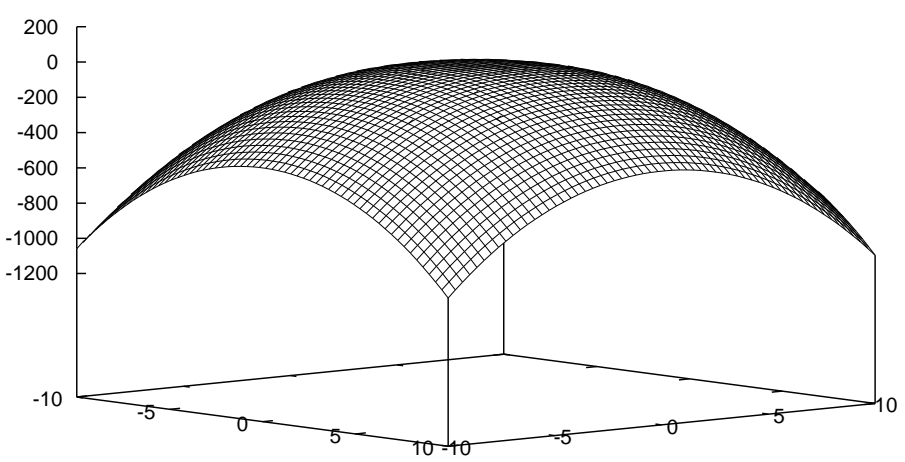

FIG. 2: The thin plate spline radial basis function $t(r)=r^{2} \ln \left(r^{2}\right)$

ref. [9], [12]), of the form

$$
t(\underline{r})=K(\underline{r})+\sum_{l=1}^{N} \lambda_{l} f\left(\underline{r}-\underline{r}_{l}\right), \quad \underline{r} \in R^{2}
$$

where $K(\underline{r})=c_{0}+c_{1} x+c_{2} y$ is the added plane. $N$ is the number of support points. The real-valued weight is characterized by $\lambda_{l} . f\left(\underline{r}-\underline{r}_{l}\right)$ is a radial basis function, a real function of positive real values depending on the distance between the grid points $\underline{r}$ and the support points $\underline{r}_{l}$.

Given the scattered data points $\underline{r}_{i}$ and the data $z_{i}=z\left(\underline{r}_{i}\right)$, the TPS satisfies the interpolation conditions

$$
t\left(\underline{r}_{i}\right)=K\left(\underline{r}_{i}\right)+\sum_{l=1}^{N} \lambda_{l} f\left(\underline{r}_{i}-\underline{r}_{l}\right)=z_{i}, \quad i=1, \ldots, N
$$

with

$$
f(\underline{r})=\underline{r}^{2} \ln \left(\underline{r}^{2}\right) .
$$

and it minimises

$$
\|t\|^{2}=I[t]=\iint_{\mathrm{R}^{2}}\left(t_{x x}^{2}+2 t_{x y}^{2}+t_{y y}^{2}\right) d x d y
$$

the 2-d case of Eq.2. $\|t\|^{2}$ is a measure of the bending energy of $t$. In other words, given a set of data points, a weighted combination of TPSs centered about each data point gives the interpolation function that passes through the points exactly while minimizing the so-called "bending energy". In order to fit the TPS to the data, it is necessary to solve for the weights and the planes' coefficients so that it is possible to compute the local TPS amplitude:

$$
t\left(\underline{r}_{i}\right)=t\left(\underline{r}_{i}, N,\left\{\underline{r}_{l}, z_{l}, l=1, \ldots, N\right\}\right)
$$

which is a function of $z_{l}$, the given emission intensity at the knot positions $r_{l}$. The TPS interpolant is defined by the coefficients, $c_{i}$ of the plane $K(\underline{r})$ and the weights $\lambda_{l}$ of the basis functions. Given the interpolation values $\underline{z}=\left(z_{1}, \ldots, z_{N}\right)$, we search for the weights $\lambda_{l}$ and $c_{i}$ so that the TPS satisfies:

$$
t\left(\underline{r}_{l}\right)=z_{l}, \quad l=1, \ldots, N
$$

and in order to have a converging integral, the following conditions need to be satisfied:

$$
\sum_{l=1}^{N} \lambda_{l}=\sum_{l=1}^{N} \lambda_{l} x_{l}=\sum_{l=1}^{N} \lambda_{l} y_{l}=0
$$


The coefficients of the TPS, $\lambda_{l}$, and the plane, $c_{i}$, can be found by solving the linear system, that may be written in matrix form as:

$$
\left(\begin{array}{ll}
\frac{F}{\underline{Q}^{T}} & \underline{\bar{Q}} \\
\underline{0}^{2}
\end{array}\right)\left(\begin{array}{l}
\underline{\lambda} \\
\underline{c}
\end{array}\right)=\left(\begin{array}{l}
\underline{z} \\
0
\end{array}\right)
$$

where the matrix components are:

$$
\begin{aligned}
& F_{i j}=f\left(\underline{r}_{i}-\underline{r}_{j}\right) \\
& \underline{z}=\left(z_{1}, \ldots, z_{N}\right)^{T} \\
& 0=(0,0,0)^{T} \\
& \underline{\lambda}=\left(\lambda_{1}, \ldots, \lambda_{N}\right)^{T} \\
& \underline{c}=\left(c_{0}, c_{1}, c_{2}\right)^{T}
\end{aligned}
$$$$
\underline{\underline{Q}}=\left(\begin{array}{ccc}
1 & x_{1} & y_{1} \\
1 & x_{2} & y_{2} \\
\vdots & \vdots & \vdots \\
1 & x_{N} & y_{N}
\end{array}\right)
$$

After having solved for $(\underline{\lambda}, \underline{c})^{T}$, the TPS can be evaluated at any point and total curvature $I[t]$ is easily accessible using the relation

$$
I[t]=\underline{\lambda}^{T} \underline{\underline{F}} \underline{\underline{\lambda}} .
$$

\section{A. The Bayesian Framework}

Once the number $N$ of support points, the location of the support points $\underline{R}=\left(\underline{r}_{1}^{T}, \ldots, \underline{r}_{N}^{T}\right)$ and the respective intensity $\underline{z}=$ $\left(z_{1}, \ldots, z_{N}\right)$ is fixed the thin-plate spline model $M$ is completely determined and fully characterized by the set $N, \underline{R}, \underline{z}$ :

$$
t(\underline{r})=t(\underline{r} \mid N, \underline{\underline{R}}, \underline{z})
$$

To compute the probability for the different models we start with Bayes theorem:

$$
p(M \mid D, I)=\frac{p(M \mid I) p(D \mid M, I)}{p(D \mid I)}=\frac{p(M \mid I) \int d \underline{z} p(D \mid M, \underline{z}, I) p(\underline{z} \mid M, I)}{p(D \mid I)} .
$$

The likelihood term $p(D \mid M, \underline{z}, I)=p(D \mid t, I)$ for $N_{d}$ measured data points is given by

$$
p(D \mid t, I)=\prod_{i=1}^{N_{d}} \frac{1}{\sqrt{2 \pi} \sigma_{i}} \exp \left(-\frac{1}{2}\left(\frac{d_{i}-O_{i}[t]}{\sigma_{i}}\right)^{2}\right),
$$

for Gaussian distributed noise with zero mean and variance $\sigma^{2}$. The function $O_{i}$ describes the weighted summation of the intensities of the thin-plate spline within the viewing cone of sensor $i$ (cf. Eq. 1). The first term in the nominator of Eq. 13 is the prior probability for the model $M$. It factors into the prior for the number of support points $N$ and the prior for the support points $p(\underline{\underline{R}} \mid N, I)$. The discretized grid for the support points provides a maximum number $N_{G}$ of possible locations (see Fig.3), which cannot be exceeded. The uninformative prior is therefore (a minimum number of 4 support points is needed to define a TPS)

$$
p(M \mid I)=p(\underline{\underline{R}} \mid N, I) p(N \mid I)=\frac{1}{\left(N_{G}-3\right)\left(\begin{array}{c}
N_{G} \\
N
\end{array}\right)} .
$$

Finally we need to assign the prior for the z-values of the support points. As testable information we use the global curvature $I[t]$ of the TPS. The maximum entropy principle yields as prior probability

$$
p(t \mid \mu, I)=\frac{1}{Z(\mu)} \exp (-\mu I[t])
$$

where we had to introduce $\mu$ as scale parameter with a Jeffreys' prior $p(\mu \mid I) \propto 1 / \mu$. However, since $t$ is completely determined by the set $N, \underline{R}, \underline{z}$ we can rewite the previous equation as prior for $\underline{z}$ :

$$
p(\underline{z} \mid \mu, M, I)=p(\underline{z} \mid \mu, N, \underline{\underline{R}}, I)=\frac{1}{Z(\mu, N, \underline{\underline{R}})} \exp (-\mu I[t(\underline{z})]) .
$$




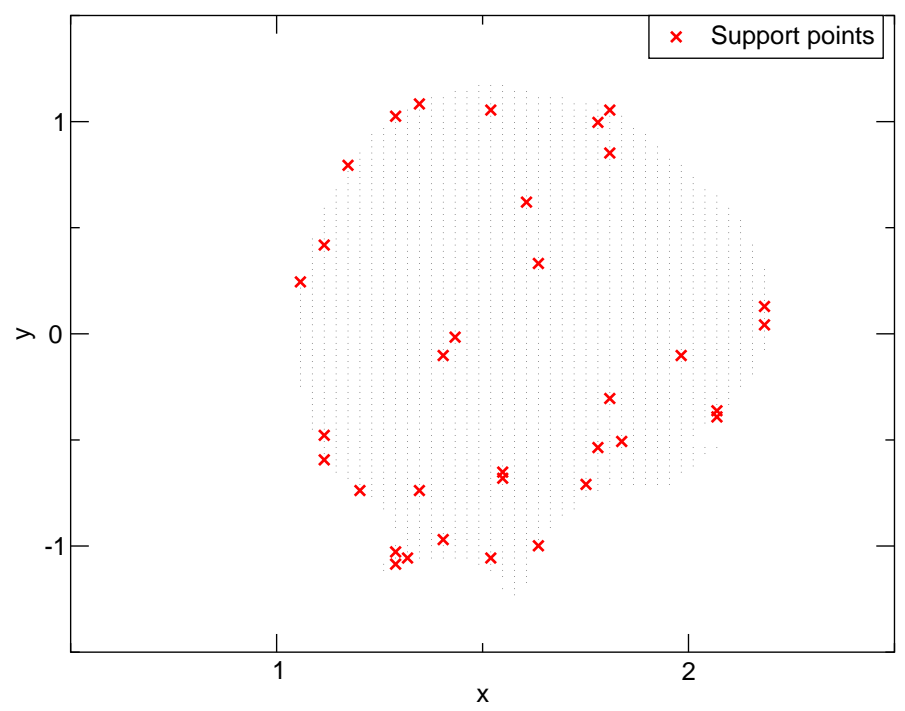

FIG. 3: The grid indicating the possible support point locations is adapted to the shape of the plasma vessel and the best distribution of the support points is indicated by stars.

The normalization $Z(\mu, N, \underline{R})$ along with the prior probability of the model M tends to keep the number of support points as small as possible. Since we use optimization algorithms to compute the most likely vector $\underline{z}$ we avoid the cumbersome marginalization of the hyperparameter (which is more easily accessible using MCMC methods) and instead look for the most likely value $\mu^{*}$ of the hyperparameter using

$$
p(\mu \mid D, M, I)=p(\mu \mid I) p(D \mid \mu, M, I)=p(\mu \mid I) \int d z p(D \mid \mu, \underline{z}, M, I) p(\underline{z} \mid \mu, M, I),
$$

followed by an approximation with a multi-variate Gaussian distribution around the maximum :

$$
p(D \mid \underline{z}, \mu, M, I) p(\underline{z} \mid \mu, M, I) \approx p\left(D \mid \underline{z}^{*}, \mu, M, I\right) p\left(\underline{z}^{*} \mid \mu, M, I\right) \exp \left\{-\frac{1}{2} \Delta \underline{z}^{T} H \Delta \underline{z}\right\}
$$

where $\Delta \underline{z}=\underline{z}-\underline{z}^{*}, \underline{z}^{*}$ being the position of the maximum of the integrand in eq. 18 , and the Hessian matrix elements $H_{i j}:=$ $-\frac{\partial^{2} \ln [p(\bar{D} \mid \underline{z}, \mu, M, I) p(\underline{z} \mid \mu, M, I)]}{\partial \underline{z}_{i} \partial \underline{z}_{j}}$. Using this approximation the integration in Eq.18 can be carried out, yielding

$$
p(\mu \mid D, M, I)=p(\mu \mid I) p\left(D \mid \mu, \underline{z}^{*}, M, I\right) p\left(\underline{z}^{*} \mid \mu, M, I\right) \sqrt{\frac{(2 \pi)^{N}}{\operatorname{det}(H)}}
$$

followed by an 1-D line scan in $\mu$ to determine $\mu^{*}$. Using $\mu^{*}$ in Eq.17 we have all the necessary quantities to evaluate Eq. 13 and to obtain the model probability $p(M \mid D, I)$. As final step simple sampling over different model orders (the number of support points is varied between 4 and $N_{G}$ ) and the locations of the support points is performed resulting in a distribution of model probabilities depending on $N$ and $\underline{\underline{R}}$.

\section{APPLICATIONS}

The TPS approach has successfully been applied to a number of different soft X-ray and bolometry tomographic measurements. Here we display mock data which closely resemble the experimental observations and the measurement uncertainties. As can be seen in Fig. 4a two high emission spots are located in the lower parts of the plasma vessel (the divertor) where the plasma touches the wall. The intensity in the main chamber varies only slightly but at the top of the plasma vessel there is an extended area, emitting slightly more than its environment. Additionally there is a hot spot visible slightly below the midplane on the right hand side. The reconstruction, displayed in Fig. $4 \mathrm{~b}$ is displaying all the key features and does not introduce any spurious ringing despite the peaked intensity profile and the edge location of most of the emission maxima. The difference between the 

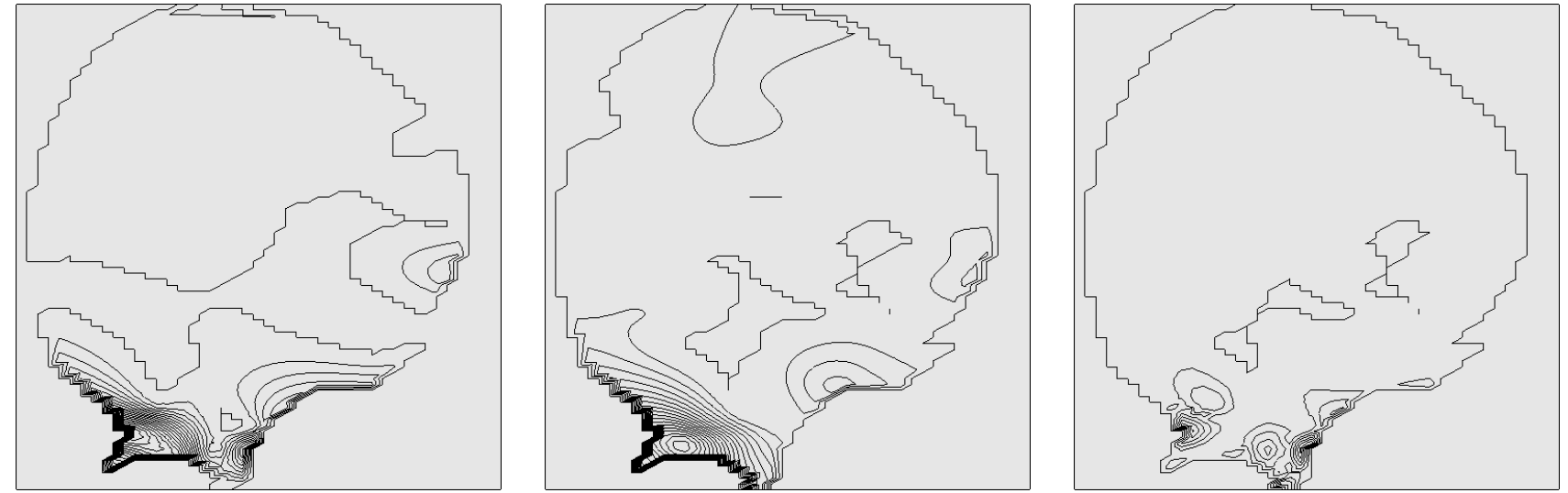

FIG. 4: a) On the left: Mock 2-D intensity profile in agreement with experimental observations b) Mid dle: reconstructed intensity profile using thin-plate splines as basis functi lute scale).

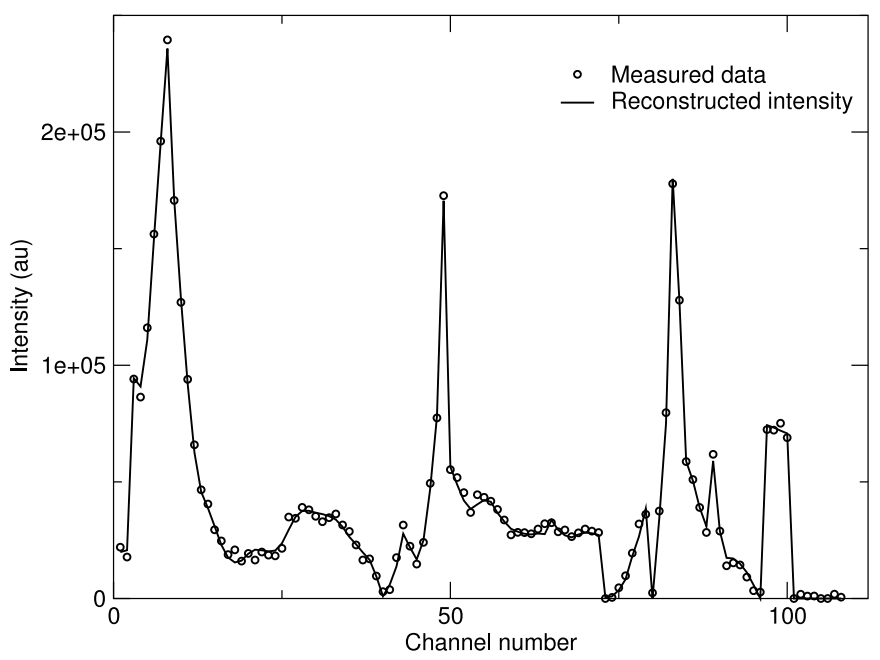

FIG. 5: Sensor data and intensities obtained from the reconstruction

mock profile and the reconstruction is mostly due to the slightly smoother reconstruction of the profiles. In Fig. 5 the measured line-integrated signal is compared to the intensity derived from the reconstructed profile. The agreement is within the statistical uncertainties without overfitting tendency. The underlying spatial distribution of the support points is displayed in Fig.3. Most support points are in or close to the divertor therefore adaptively enhancing the flexibility of the reconstruction where the profile has the largest variations.

\section{SUMMARY}

We presented a Bayesian analysis for the 2-d profile reconstruction from line-integrated chord measurements which augments the usual approaches by the concept of data dependent adaptive resolution and local smoothness. All structures in the reconstruction are supported by data constraints. This has been demonstrated by a mock bolometry example with realistic uncertainties. The straightforward next step is the addition of a suitable MCMC-method (see [13]) to improve on the presently used evidence approximations and the integration into the framework of Bayesian Neural Networks [14] to achieve real-time deconvolution capabilities with $\approx 10^{3}$ deconvolutions/s.

[1] Radon J., Berichte Sächsische Akademie der Wissenschaften 69,262 (1917). 
[2] Deans S., The Radon Transform and Some of its applications, Wiley, New York (1983).

[3] Granetz R. S., Smeulders P., Nucl. Fusion 28, 457 (1988).

[4] Cormack M. A., J. Appl. Phys. 35, 2908 (1964).

[5] Fuchs G., Miura Y., Mori M., Plasma Phys. Control. Fusion 36, pp. 307-316 (1994).

[6] Boivin R. L., Goetz J. A., Marmar E. S., Rice J. E., Terry J. L., Rev. Sci. Instr. 70(1), pp. 260-264 (1999).

[7] Ertl K., von der Linden W., Dose V., Weller A., Nucl. Fusion 36(11), pp.1477-1488 (1996).

[8] Golan A., Dose V., J.Phys.A.,34, pp.1271-1283 (2001).

[9] Wahba G., Spline Models for Observational Data, Society for Industrial and Ap plied Mathematics, Pennsylvania (1990).

[10] von der Linden W., Dose V., Fischer R., Proceedings of the Maximum Entropy Conference 1996, M. Sears, V. Nedeljkovic, N.E. Pendock, S. Sibisi, eds., 154 (1996).

[11] Guglielmetti F., Fischer R., Dose V., Proceedings of the Maximum Entropy Conference 2004, R. Fischer, R. Preuss and U. von Toussaint, eds., AIP 735, p. 111-118 (2004).

[12] Meinguet J., J.Appl.Math.Phys. (ZAMP), 30, pp.292-304 (1979).

[13] Preuss R., von Toussaint U., Proceedings of the Maximum Entropy Conference 2007 (these proceedings).

[14] von Toussaint U., Gori S., Dose V., Neural Networks 19(10), pp.1550-1557 (2006). 